\title{
Infrared pyrometer for temperature measurement of objects, emissivity of which depends on wavelength and time
}

\author{
by Z. Bielecki ${ }^{1}$, K. Chrzanowski ${ }^{1}$, R. Matyszkiel ${ }^{3}$, T. Piątkowski ${ }^{1}$
}

and M. Szulim ${ }^{2}$

${ }^{1}$ Military University. of Technology, Institute of Optoelectronics, 01-489 Warsaw, Poland

${ }^{2}$ Military University of Technology, Institute of Fundamentals of Electronics, 01-489 Warsaw, Poland

${ }^{3}$ Military Institute of Communication, 05-130 Zegrze PId., Poland; tel 48-22-6859789

fax 48-22-6668950, e-mail:zbieleck@wat.waw.pl., kchrza@wat.waw.pl

\begin{abstract}
The multiband MBP 98A pyrometer developed for non-contact temperature measurement of objects with unknown and wavelength-dependent emissivity has been presented in this paper. The pyrometer was designed using single thermoelectrically cooled $\mathrm{PbS}$ detector of spectral band of 1-2.5 $\mu \mathrm{m}$ and 8 narrow-band optical fitters. It enables temperature measurement of objects at temperature within a range of $500^{\circ} \mathrm{C}-1200^{\circ} \mathrm{C}$ and a speed of $75 \mathrm{~Hz}$. It can be used for controlling various industrial-technological processes as well as in research works for testing of classic singleband pyrometers in temperature measurement of objects whose emissivity depends on the wavelength and time.
\end{abstract}

\section{Introduction}

A division of systems for non-contact temperature measurements employing radiation emitted by a tested object is based on a number of spectral bands used by the measuring system. There are three groups of systems currently in use: singleband, dualband, and multiband ones. The singleband systems determine the object temperature on the basis of the signal measured in one spectral band; for dual systems - in its two spectral bands, and for multiband systems - in at least three bands.

Nowadays, not less than $90 \%$ of systems in the market are singleband systems; dualband systems are rather infrequent; the multiband systems are still at laboratory stage of development. However, an increasing interest in multiband systems can be noted $[1,2,3,4,6]$ as they can potentially bring significant accuracy improvement in non-contact temperature measurements, particularly in the case of so-called "difficult objects". These objects are considered to be the ones whose emissivity depends on the wavelength and time. Such cases can be found in many industrial applications and particularly often in the steel and semiconductor industry.

This paper presents the development results of the multiband pyrometer for a noncontact temperature measurement of the objects with unknown and wavelength-dependent emissivity. The errors of temperature measurements of such objects using classic singleband systems are often relatively high. Moreover, it is very difficult to estimate such errors.

A review of infrared systems for non-contact temperature measurement, using radiation emitted by the tested object, is presented in Section 2 . The basic concept of the developed pyrometer is shown in Section 3. The design details of an experimental MBP 98 A multiband pyrometer developed in the Institute of Optoelectronics of the Military University of Technology are presented in Section 4. Results of its testing are demonstrated in Section 5 , and final conclusions in Section 6. 


\section{Division of IR systems for non-contact temperature measurement}

Singleband systems enable direct measurement of power of radiation emitted by the tested object in a single spectral band. Radiation emitted by the object that reaches detector produces a signal at detector output. The value of this signal carries information about object temperature, and the latter parameter is usually determined using the system calibration chart. Measurement method requires knowledge about object emissivity. Inaccurate estimation of this parameter is the most common source of significant errors in temperature measurement for singleband systems

Dualband systems measure radiation in two separate spectral bands. Object temperature is usually determined by using the system calibration chart that presents the inter-relationships of the signals measured in these two bands on the object's temperature. However, their indications still depend on the object's emissivity in applications where the non-grey-body objects are tested.

The multiband systems apparently differ from the single- or dualband systems only because of the higher number of system's spectral bands. However, the differences are much more significant. Single- or dualband systems usually use their calibration chart or a single analytical formula for temperature determination. Multiband systems determine object temperature by solving a set of $n$ equations with $m$ unknowns, as presented below:

$$
\begin{aligned}
& S_{1}=f\left(T_{o b}, \varepsilon\left(\lambda_{1}\right)\right), \\
& S_{2}=f\left(T_{o b}, \varepsilon\left(\lambda_{2}\right)\right), \\
& S_{n}=f\left(T_{o b}, E\left(\lambda_{n}\right)\right),
\end{aligned}
$$

where $n$ is the number of detection bands, $S_{n}$ is the signal measured at $n$ band, $T_{o b}$ is the real object's temperature, $\delta(\lambda)$ is the object emissivity at wavelength $\lambda$.

If the number of system's spectral bands $n$ is higher than the number of the unknowns $m$ of the theoretical model, then it is possible to solve the set of equations (1) and to determine the object temperature $T_{o b}$. The different values of the object emissivity for different spectral bands are the main obstacle to obtain the number of system spectral bands equal to the number of the unknowns. The system closure can be achieved by setting equal emissivities in certain pairs of spectral bands [1]. Other methods include the so-called balancing of intermediation observation [2] and curve fitting of spectral emissivity [3].

\section{The basic concept of the developed MBP 98A pyrometer}

As it was mentioned earlier the system closure of the set of equations (1) can be achieved using different methods. Any of these methods enable solving the set of the equations (1) and determination of the object temperature. However, on the basis of the reports on practical multiband systems [4] and patent analysis it seems that the recent method presented in this report can be commonly accepted as the standard one for the multiband systems. Therefore, it has been decided to design a system with application of the method of curve fitting of spectral emissivity and to assume that the object emissivity can be always presented in the following form

$$
\varepsilon_{o b}(\lambda)=a_{0}+a_{1} \lambda+a_{2} \lambda^{2}+\ldots .+a_{n-2} \lambda^{m-2}
$$

where $m$ must be always not higher than the number of spectral bands.

With this assumption for the object emissivity there are at least $m$ unknowns ( $m-1$ polynomial coefficients plus an object temperature) in the set of equation (1). On the basis of the analysis of emissivity curves of objects in industrial applications the assumption was made that emissivity curves of such objects can be well interpolated using polynomials of the degree not higher than 2 [7]. As it can be seen in Fig.1 the possible emissivity curves 
interpolated by polynomials of 2 -th degree are close to emissivity curves of many materials used in industry.

The errors of temperature measurement with multiband systems caused by a detector noise and other internal disturbances decrease when the difference between the number of system spectral bands $n$ and the number of the unknowns $m$ rise [8]. Therefore, the system of eight spectral bands has been designed; i.e., two times higher number of bands $n$ than the number of unknowns $m$.

For the system of $n=8$ and the object of emissivity interpolated with polynomials of degree not higher than 2 we have to solve the following set of equations

$$
\begin{aligned}
& S_{1}\left(T_{o b}\right)=\left(a_{0}+a_{1} \lambda_{1}+a_{2} \lambda_{1}{ }^{2}\right) S_{b b}\left(T_{o b}, \lambda_{1}, \Delta \lambda_{1}\right) ; \\
& S_{8}\left(T_{o b}\right)=\left(a_{0}+a_{1} \lambda_{8}+a_{2} \lambda_{8}{ }^{2}\right) S_{b b}\left(T_{o b}, \lambda_{8}, \Delta \lambda_{8}\right),
\end{aligned}
$$

where $S_{n}$ is the signal at the output of the detector from the real object at $n$ spectral band, $\lambda_{n}$ is the effective centre of the $n$ spectral band, $\Delta \lambda_{n}$ is the effective width of the $n$ band, $T_{o b}$ is the object or blackbody temperature and $S_{b b}$ is the signal caused by the radiation emitted by a blackbody for the calibration condition equal to

$$
S_{h b}\left(T_{o h}, \lambda_{n}, \Delta \lambda_{n}\right)=k M\left(T_{o h}, \lambda_{n}\right) \tau_{o}\left(\lambda_{n}\right) \tau_{k}\left(\lambda_{n}\right) s\left(\lambda_{n}\right) \Delta \lambda_{n} d \lambda
$$

where $M\left(T_{o b}, \lambda_{n}\right)$ is the spectral existence at the temperature $T_{n}$ and the wavelength $\lambda_{n}$ and $k$ is the constant influenced by the optics $F$ number, the detector sensitivity and electronics gain that can be determined experimentally during the calibration process.

Finally, we have a new set of equations

$$
\begin{gathered}
S_{1}=k_{1}\left(a_{0}+a_{1} \lambda_{1}+a_{2} \lambda_{1}^{2}\right) s\left(\lambda_{1}\right) \tau_{F 1} \tau_{o}\left(\lambda_{1}\right) \Delta \lambda_{1}\left\{\lambda_{1}^{5}\left[\exp \left(\frac{c_{2}}{\lambda_{1} T_{o b}}\right)-1\right]\right\}^{-1} \\
S_{8}=k_{8}\left(a_{0}+a_{1} \lambda_{8}+a_{2} \lambda_{8}^{2}\right) s\left(\lambda_{8}\right) \tau_{F 2} \tau_{o}\left(\lambda_{8}\right) \Delta \lambda_{8}\left\{\lambda_{8}^{5}\left[\exp \left(\frac{c_{2}}{\lambda_{8} T_{o b}}\right)-1\right]\right\}^{-1} .
\end{gathered}
$$

where $\tau_{0}$ is optics transmittance, $\tau_{F}$ is filter transmittance, $s(\lambda)$ is detector relative responsivity.

The set of equations (5) cannot be solved analytically. It can be solved only by means of various numerical methods. The least squared method was chosen for the MBP 98A pyrometer.

Using the above mentioned method, the unknown parameters $T_{o b}, a_{0}, a_{1}, a_{2}$ can be determined by finding the global minimum of the function $I s m\left(T_{o b}, a_{0}, a_{1}, a_{2}\right)$ presented below

$$
l s m\left(a_{0}, a_{1}, . ., a_{2}, T_{o h}\right)=\sum_{i=1}^{8}\left\{S_{i}-\frac{k_{i}\left(a_{0}+a_{1} \lambda_{i}+a_{2} \lambda_{i}^{2}\right) s\left(\lambda_{i}\right) \tau_{o}\left(\lambda_{i}\right) \tau_{F i} \Delta \lambda_{i}}{\lambda_{i}^{5}\left[\exp \left(\frac{c_{2}}{\lambda_{i} T_{o b}}\right)-1\right]}\right\}^{2}
$$

The fast algorithm enabling to solve the equation (6) was developed. Next, it was implemented in a software package that made it possible to determine an object's temperature during real time measurements.

\section{Design of the MBP 98A pyrometer}

The block diagram of the MBP 98A pyrometer is presented in Fig.2. A principle of operation of the pyrometer is as follows. 
An infrared radiation from the tested object is focused on a photoconductive infrared detector of the PbS type using BK 7 glass - silica achromatic type optical system on photoconductive type, PbS infrared detector. The optical system was optimised to have the aberration blur smaller than the diameter of the detector. Moreover, the optical system is characterised by a small F-number that enables to obtain a high signal-to-noise ratio.

A signal from the object is modulated by a rotary plate on which eight optical filters are fitted. Spectral bands of most of the filters were chosen to minimise an influence of atmospheric absorption on a signal from the object to the detector.

The detector absorbs infrared radiation with the wavelengths shorter than its cut-off wavelength. Absorption of such radiation causes an increase in electrical conductivity of materials and corresponding decrease in the detector resistance. This effect enables us to measure radiation reaching the detector. However, the resistance is changed only by a small fraction, typically by less than $1 \%$.

A photoconductive type PbS infrared detector of a spectral band 1-2.5 $\mu \mathrm{m}$ was chosen for application in the pyrometer due to several factors. First, it was noticed from the simulations that $1-2.5 \mu \mathrm{m}$ is an optimum spectral band for the required temperature measurement range. Second, low-cost materials can be used to design an optical system for $1-2.5 \mu \mathrm{m}$ spectral band. Third, this type of IR detectors is characterised by a relative low price in comparison to HgCdTe detectors.

Two-stage thermoelectric cooler that ensures a detector temperature about $-25^{\circ} \mathrm{C}$, when the ambient temperature is about $+25^{\circ} \mathrm{C}$, is used in order to increase detector sensitivity. The thermoelectric cooler is biasing by subminiature proportional temperature controller of the HY-5600 type, of the HYTEC firm. This device is intended for "cool only" fixed temperature applications where the front panel controls and digital readouts are not required. The HY5600 operates in conjunction with a thermistor bridge to precisely measure and regulate the temperature of a device affixed to the TEC with resolution below $0.1 \mathrm{~K}$. The Th thermistor is located as close to the TEC as possible in order to avoid a thermal lag.

A preamplifier is used to amplify a very small signal at the output of the $\mathrm{PbS}$ detector. The preamplifier is characterised by a low noise and ultra low input current. Total gain of this preamplifier can be set as high as $1000 \mathrm{~V} N$. The preamplifier has typical gain-bandwidth products from $\mathrm{DC}$ to $20 \mathrm{kHz}$. The signal from the output of the preamplifier is sent both to the amplifier in the main measurement channel and to an additional analogue output. The amplified analogue signal from the amplifier is next converted to a digital 12-bit word by IC MAX 199 type converter. The signal after digitisation is registered in a computer memory.

All functions of the pyrometer are controlled by a microprocessor system, single chip computer 80535 of the Siemens company. This microprocessor system groups and processes data from each filter. Next, the information is sent to a microcomputer system. An interface between a microprocessor system and a slot RS 232 of a personal computer is performed by MAX 232. Additionally, the $\mu \mathrm{P}$ system controls the driver of DC motor. LCD display allows us to present the measurements data.

Rotation of a plate is assured by DC motor, which is controlled by a driver and microprocessor system of 80535 type. A speed of rotation was optimized to have a signal from the object modulated with frequency of $600 \mathrm{~Hz}$.

$\mathrm{CW}$ laser module is used as an indicator, which allows an operator to specify a place of temperature measurement. The module is controlled by a microprocessor system.

\section{Testing results}

As the authors of this paper did not have a selective body of precisely controlled temperature and known emissivity, a blackbody was used as the reference object of temperature measurement. However, the blackbody was treated as object of unknown emissivity to simulate real measurement mode and task of system was to determine not only object temperature $T_{\text {ob }}$ but also emissivity coefficients $a_{0}, a_{1}, a_{2}$. 
Results of temperature measurement of this blackbody with the developed multiband pyrometer are shown in Fig.3. As we can see in this figure, the pyrometer enabled temperature measurement of the reference object with relative errors below level of $\pm 0.5 \%$.

\section{Conclusions}

An experimental 8-band pyrometer for non-contact temperature measurements of the objects with unknown and wavelength-dependent emissivity was developed. It enables temperature measurement of the objects with a temperature of $500^{\circ} \mathrm{C}-1200^{\circ} \mathrm{C}$. with a speed of $75 \mathrm{~Hz}$. It was experimentally verified that errors of temperature measurement of the reference object with the developed pyrometer are below level of $\pm 0.5 \%$. We can estimate that accuracy temperature measurement of other objects, whose emissivity can be exactly interpolated by a polynomial of 2-th degree, will be of similar level. It can be used for control of various industrial-technological processes, in research works, for control of thermal imagers indications as well as for control of classic singleband pyrometers.

This research was performed under the support of the State Committee for Scientific Research of Republic of Poland. The program No 8T11B 04012.

\section{REFERENCES}

[1] TANK (V.) - Infrared temperature measurement with automatic correction of the influence of emissivity, Infrared Phys. 29, 211-212 (1989).

[2] TANK (V.), DIETL (H.) - Multispectral Infrared Pyrometer For Temperature Measurement With Automatic Correction of The Influence of Emissivity, Infrared Phys. 30, 331-342 (1990).

[3] HUNTER (G. B.) et al. - Multiwavelength pyrometry: an improved method, Opt. Eng., 24, $1081-1085(1985)$.

[4] KOSONOCKY (W. F.), KAPLINSKY (M. B.), MCCAFFREY (N. J.) - Multi-Wavelength imaging pyrometer, SPIE Vol. 2225, 26-43 (1994).

[5] BARANI (G.), TOFANI (A.) - Comparison of some algorithms commonly used in infrared pyrometry: computer simulation, SPIE 1467, 458-468" (1991).

[6] KHAN (N. A.), ALLEMAND (C.), EAGAR (T. W.) - Non-contact temperature measurement: least squares based techniques, Rev, Sci. Instrum., 62, (1991).

[7] SALA (A.) - Radiant properties of materials, PWN-Polish Scientific Publishers Warsaw \& Elsevier Amsterdam-Oxford (1986).

[8] CHRZANOWSKI (K.), SZULIM (M.) - A measure of influence of detector noise on temperature measurement accuracy with IR systems, Appl. Opt., 37, 3566 (1998).

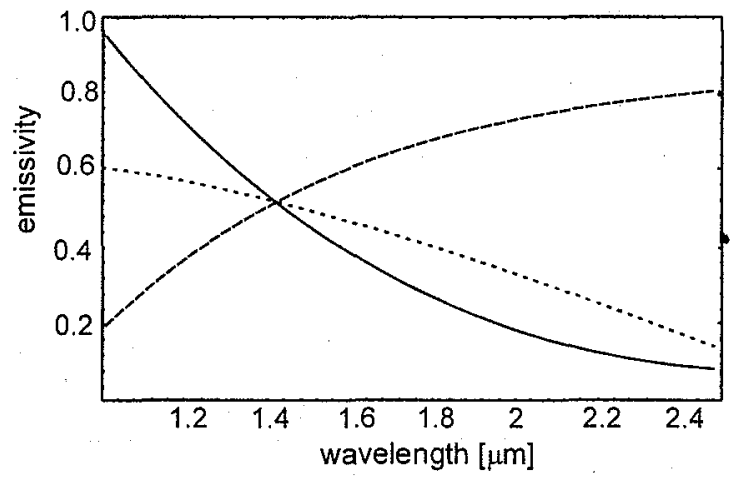

Fig.1. Examples of emissivity curves interpolated by 2-th degree polynomials 
http://dx.doi.org/10.21611/qirt.1998.050

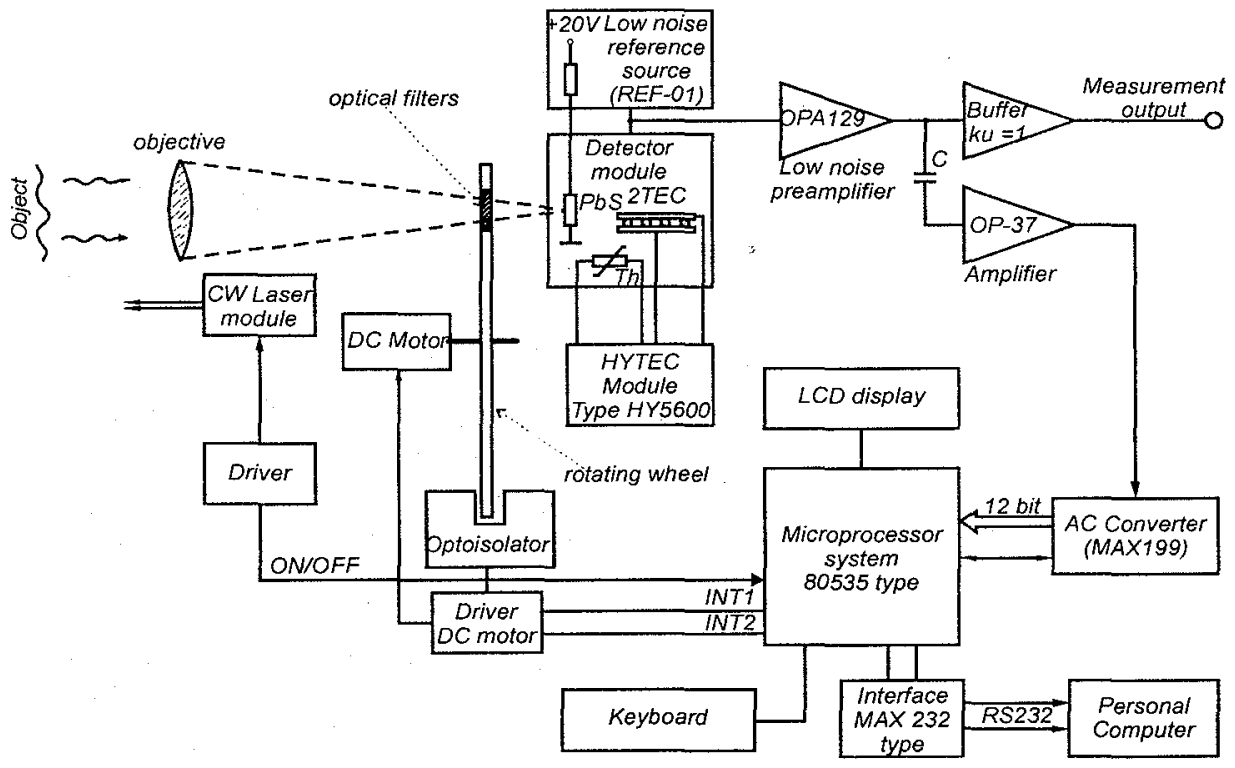

Fig. 2. Diagram of the designed pyrometer

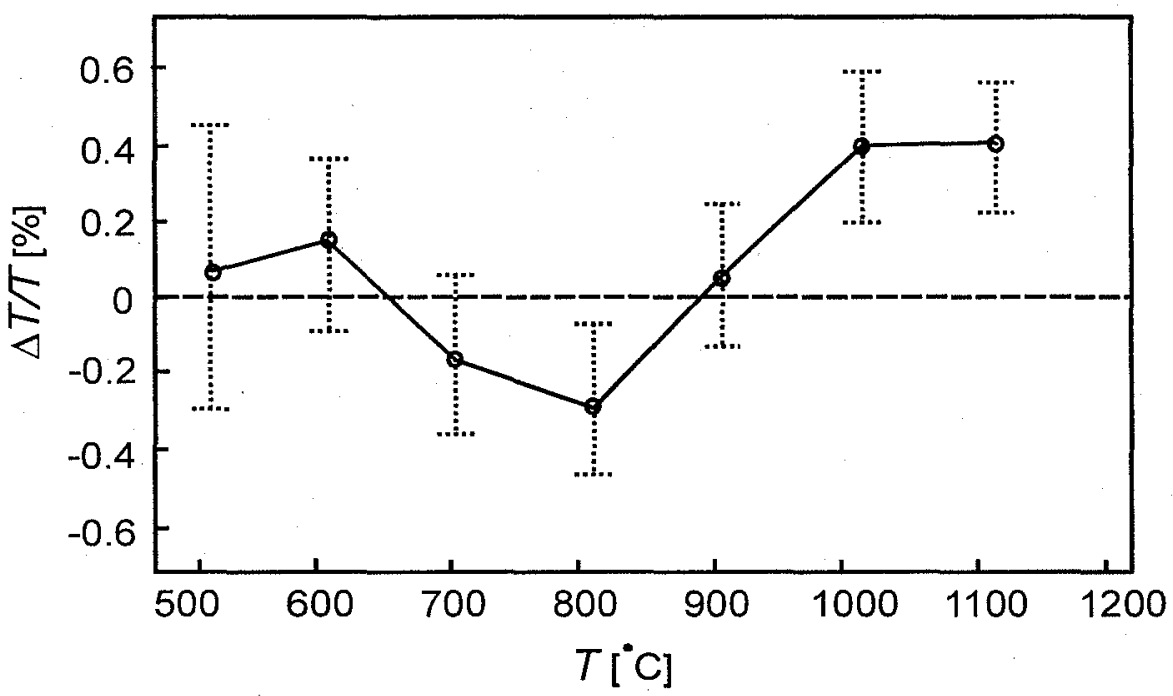

Fig. 3. Relative errors of temperature measurement of the reference object 